\title{
Características del menor y de la madre asociadas a la neumonía en niños menores de cinco años
}

\author{
Characteristics of the child and the mother associated with pneumonia in \\ children under five years of age
}

\author{
Norma Ortiz-Lizana ${ }^{1}$ \\ Katherine Ortiz-Romaní ${ }^{2}$
}

\begin{abstract}
Resumen
Objetivo: determinar la asociación entre las características del menor y de la madre y la neumonía en niños menores de cinco años. Materiales y métodos: estudio retrospectivo de casos y controles en 293 niños menores de cinco años. Los casos fueron 114 niños con neumonía y los controles fueron 130 niños sin neumonía hospitalizados en una clínica de Lima. Para el análisis bivariado se emplearon las pruebas de Chi-cuadrado, $t$ de Student y el modelo no ajustado con Odds Ratio e intervalos de confianza al $95 \%$. Resultados: un factor protector de la neumonía fue brindar lactancia materna exclusiva (OR: $0.5 ; p=0.05)$ y los factores de riesgo para presentar neumonía fueron la ocupación laboral de la madre (OR: $1.4 ; p=0.02)$, tener edades entre cuatro a cinco años (OR: 2.2; $p=0.02$ ) y tener una hospitalización (OR: 1.8; $p=0.03$ ) y enfermedad respiratoria previa (OR: 3.8; $p=0.002$ ). Conclusión: brindar lactancia materna exclusiva es un factor protector de la neumonía. Mientras que la ocupación laboral de la madre, las edades entre cuatro a cinco años y tener una hospitalización y enfermedad respiratoria previa del menor son factores de riesgo para pre sentar neumonía en niños menores de cinco años. Ante estos hallazgos se incentiva que el personal de enfermería tome en cuenta los mismos para brindar una adecuada campaña de prevención sobre la neumonía a las madres y cuidadores, promocionando la práctica de lactancia materna exclusiva.
\end{abstract}

Palabras clave: Neumonía; Lactancia Materna; Salud del Niño (Fuente: DeCS).

\begin{abstract}
Objective: to determine the association between the characteristics of the child and pneumonia in children under five years of age. Materials and methods: a retrospective study of cases and controls in 293 children under five years of age. The cases were 114 children with pneumonia and the controls were 130 children without hospitalized pneumonia in a clinic in Lima. For the bivariate analysis, Chi-square, the student and the unadjusted model were used with the probability ratio and $95 \%$ confidence intervals. Results: one pneumonia protection factor was exclusive breastfeeding (OR: $0.5 ; \mathrm{p}=0.05)$ and risk factors for presenting pneumonia in the mother (OR: 1.4, $\mathrm{p}=0.02)$ four to five years (OR: 2.2, $\mathrm{p}=0.02$ ) and have a hospitalization (OR: $1.8, \mathrm{p}=0.03$ ) and previous respiratory disease (OR: $3.8, \mathrm{p}=0.002)$. Conclusion: providing exclusive breastfeeding is a protective factor of pneumonia. While the occupation of the mother, ages between four and five years and having a hospitalization and a previous respiratory disease are risk factors for pneumonia in children under five years. The search for information on pneumonia for mothers and caregivers, promoting the practice of exclusive breastfeeding.
\end{abstract}

Keys words: Pneumonia; Breast Feeding; Child Health (Source: DeCS).

Para citar:

Ortiz N, Ortiz K. Características del menor y de la madre asociadas a la neumonía en niños menores de cinco años CASUS. 2018;3(3):138-145.

${ }^{1}$ Clínica Mundo Salud. Bachiller de Enfermería.

${ }^{2}$ Universidad Católica Sedes Sapientiae. Licenciada de Enfermería. Correo electrónico: nortizlizana@gmail.com
Fecha de recepción: 03-10-18

Fecha de envío a pares: 04-10-18

Fecha de aprobación por pares: 01-12-18

Fecha de aceptación: 07-12-18 


\section{INTRODUCCIÓN}

La neumonía es un tipo de infección respiratoria aguda (IRA) que daña a los pulmones (1) causada principalmente por agentes patógenos como el Streptococcus pnuemoniae, el Haemophilus influenzae tipo b y el virus sincitial respiratorio (2). Además, se caracteriza por presentar disnea, fiebre, tos, pérdida del apetito, taquipnea y tiraje subcostal. Actualmente se le considera como un problema para la salud pública e infantil a nivel mundial $(3,4)$.

La Organización mundial de la Salud (OMS) afirmó que en el 2015 la neumonía es la principal causa de mortalidad infantil y responsable del 15 $\%$ de todas las defunciones en menores de 5 años y se calcula que mato a unos 920136 niños $(3,4)$. Por otro lado, la incidencia a nivel mundial de esta patología en este grupo de edad fue de 156 millones de episodios, de los cuales 151 millones se registran en países en desarrollo. Existe una mayor prevalencia, incidencia y mortalidad de esta enfermedad en África subsahariana y Asia meridional. $\mathrm{Y}$ de los casos de incidencia entre un $7 \%$ a $13 \%$ son muy graves poniendo en peligro la vida del niño y requieren hospitalización $(2,3)$. A nivel nacional la mortalidad por casos de neumonía en los niños ha disminuido gracias a las campañas de inmunizaciones (5). Sin embargo, la tasa de letalidad aún se mantiene en niveles similares (6).

Considerando que la mayoría de casos de neumonía ocurren en niños menores de cinco años es importante identificar los factores de riesgo que pueden repercutir en el desarrollo de esta patología para poder prevenirla. Lamentablemente aún sigue siendo una causa constante de morbi-mortalidad a nivel mundial y nacional (5-7). Además, se debe tomar en cuenta que los niños en edades preescolares por sus condiciones fisiológicas y las características de sus mecanismos defensivos (inmadurez del sistema inmunitario) presentan predisposición de infecciones respiratorias (8). Esto se agravaría aún más si el niño tuviese una enfermedad previa o un descuido por parte de su madre en la alimentación durante sus primeros meses de vida (9).
Respecto a la alimentación, los niños que no recibieron lactancia materna exclusiva (LME) tienen probabilidades de contraer e incluso morir por diversas patologías que afectan a los pulmones $(9,10)$. La leche materna es considerada un alimento que contiene factores inmunológicos que previene enfermedades infecciosas en la población infantil, aunque no es brindada por todas las madres $(10,11)$. En el Perú solo el $66.1 \%$ de las madres manifestó brindar LME a sus hijos, encontrando un mayor porcentaje en el área rural $80.3 \%$ en comparación con el área urbana (59.9 $\%)(8,12)$. Además, actualmente las madres utilizan sólo fórmulas para alimentar a sus hijos menores de 6 meses por motivos laborales, estéticos, falta de capacitación sobre la importancia de LME por un personal de salud (teniendo en cuenta que el personal de enfermería cumple un rol importante en la prevención de la neumonía y otras enfermedades respiratorias), etc. (13) Cabe destacar que las fórmulas solo deberían ser empleadas para casos excepcionales $(14,15)$.

Frente a esta problemática que afecta la salud de los niños menores de cinco años y considerando que la neumonía sigue siendo una de las principales causas de muertes tanto a nivel internacional y nacional $(16,17)$ es de suma importancia investigar no sólo las características del niño que tiene neumonía, sino las características de la madre como principal cuidador del menor (16). Para el personal de enfermería le ayudaría a precisar intervenciones adecuadas de promoción y prevención de esta patología dirigidas a las madres u otros cuidadores de los menores de edad. Por esta razón, el objetivo del estudio fue determinar la asociación entre las características del menor y de la madre y la neumonía en niños menores de cinco años.

\section{MATERIALES Y MÉTODOS}

Se realizó un estudio retrospectivo de casos y controles con alcance correlacional y explicativo. La muestra del estudio fue de 293 niños menores de cinco años que se atendieron en una clínica de Lima Norte en el periodo de enero a setiembre 2018, con los que se formaron dos grupos (ver 
figura 1): grupo de casos (niños menores de cinco años con neumonía) estuvo conformado por 134 niños con neumonía extrahospitalaria. Grupo controles (niños menores de cinco años sin neumonía) estuvo conformado por 159 niños menores de cinco años.

Figura 1. Diagrama de flujo para determinar la muestra

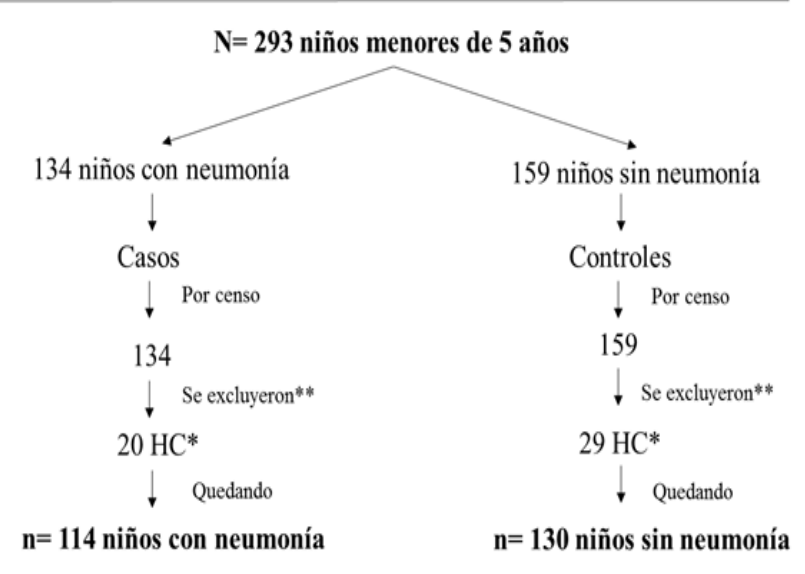

* HC: Historias clinicas

** Se excluyeron las HC de los lactantes menores de 6 meses

Se excluyeron las historias clínicas con datos incompletos y a niños menores de 6 meses, porque se quiso medir la variable lactancia materna exclusiva durante los 6 meses.

Para la recolección de datos se revisó el registro de Excel diario de niños hospitalizados en servicio de pediatría durante enero a setiembre 2018, con el fin de obtener los datos del pediátrico $\mathrm{y}$ posteriormente para poder acceder al sistema virtual de las historias clínicas de los pacientes hospitalizados. A través de las mismas se identificaron las características de la madre y las características del niño agrupados de la siguiente manera:

Características madre: edad, variable cuantitativa discreta (años); lactancia materna exclusiva, variable dicotómica (sí y no); ocupación actual, variable dicotómica (sí trabaja, no trabaja); paridad, variable categórica politómica ordinal (primigesta, secundigesta y multigesta); edad gestacional, variable cuantitativa discreta (en semanas) y tipo de parto, variable dicotómica (normal, cesárea). No se consideró el tipo de seguro de salud, porque todas las madres tenían un seguro privado.

Características de los niños: edad, variable cualitativa ordinal ( $\leq 1$ año, 2-3 años y 4-5 años); hospitalización previa, variable dicotómica (sí y no); enfermedad respiratoria previa, variable dicotómica (sí y no), esta variable surgió de la causa de la hospitalización previa y que la categoría "sî" está conformada por enfermedades respiratorias como el asma, bronquitis, síndrome de obstrucción bronquial (SOB) y coqueluchoide; sexo, variable dicotómica (masculino y femenino); vacunas, variable dicotómica (completas e incompletas), para la categoría "incompleta" se tomó en cuenta si al niño le faltaba alguna vacuna; retraso de desarrollo psicomotriz, variable dicotómica (sí y no).

Para el plan de análisis se empleó el programa STATA versión 14. En el análisis bivariado descriptivo, se empleó la prueba estadística Chicuadrado y t de Student. Para determinar la asociación entre cada variable independiente y la variable dependiente se calculó con Odds Ratio e Intervalos de Confianza (IC) al 95\% con un nivel de significancia de $p \leq 0.05$.

El presente estudio fue revisado y aprobado por un comité de ética de investigación para la protección de los datos de los sujetos involucrados en el estudio.

\section{RESULTADOS}

Del total de los 293 niños menores de cinco años registrados se evidenció que el $45.7 \%$ presentó neumonía. En la tabla 1, respecto al análisis descriptivo bivariado entre las características de la madre asociadas a la neumonía en niños menores de cinco años, se muestra que la lactancia materna exclusiva $(p=0.04)$ y la ocupación laboral $(p=$ $0.01)$ se asociaron significativamente con la neumonía. Es decir no brindar LME al menor (49 $\%)$ y tener una ocupación laboral (50.6 \%) se asociaron con la neumonía de sus hijos. El resto de asociaciones no fueron significativas. En el modelo de Odds Ratio Crudo las características de la madre que se asociaron estadísticamente con la 
Tabla 1. Análisis bivariado entre las características de la madre asociados a la neumonía en niños menores de 5 años de una clínica de Lima, 2018

\begin{tabular}{|c|c|c|c|c|c|}
\hline Factores & $\begin{array}{l}\text { Casos } \\
\text { n (\%) }\end{array}$ & $\begin{array}{c}\text { Controles } \\
\text { n (\%) }\end{array}$ & p & OR $(\operatorname{IC~} 95 \%)^{\dagger}$ & p \\
\hline Edad (media \pm DE) & $28.9 \pm 3.8$ & $29.2 \pm 4.1$ & $0.70^{*}$ & $0.9(0.9-1.1)$ & 0.69 \\
\hline Lactancia materna exclusiva & & & 0.04 & & \\
\hline No & $26(49)$ & $27(51)$ & & Referencia & - \\
\hline Sí & $87(45)$ & $102(55)$ & & $0.5(0.2-1.3)$ & 0.05 \\
\hline Ocupación laboral & & & 0.01 & & \\
\hline No & $39(41.4)$ & $55(58.6)$ & & Referencia & - \\
\hline Si & $44(50.6)$ & $72(49.4)$ & & $1.4(0.8-2.4)$ & 0.02 \\
\hline Paridad & & & 0.31 & & \\
\hline Primigesta & $64(48.9)$ & $67(51.2)$ & & Referencia & - \\
\hline Secundigesta & $41(48.8)$ & $43(51.2)$ & & $0.9(0.6-1.7)$ & 0.99 \\
\hline Multigesta & $9(33.3)$ & $18(66.7)$ & & $0.5(0.2-1.2)$ & 0.15 \\
\hline Edad gestacional & $38.2 \pm 1.8$ & $38.3 \pm 1.7$ & 0.58 & $0.9(0.8-1.1)$ & 0.59 \\
\hline Tipo de parto & & & 0.14 & & \\
\hline Normal & $54(52.4)$ & $49(47.6)$ & & Referencia & - \\
\hline Cesárea & $59(42.8)$ & $79(57.2)$ & & $0.7(0.4-1.1)$ & 0.14 \\
\hline
\end{tabular}

* A partir de la Prueba de T de Student

† OR: Odss Ratio. IC: Intervalo de confianza. Los cálculos se realizaron con la categoría "casos" de la variable dependiente

neumonía fueron: lactancia materna exclusiva ( $p=0.05)$ y ocupación laboral (0.02). Los niños que recibieron LME tienen menos posibilidad de presentar neumonía en comparación con los que no recibieron LME (OR: 0.5; IC $95 \%$ : 0.2-1.3). Mientras que los niños que tienen madres con una ocupación laboral tienen 0.4 veces más de presentar neumonía que aquellos con madres que no trabajan (OR: 1.4; IC $95 \%$ : 0.8-2.4).

En la tabla 2, en el análisis bivariado se evidenció que las características del menor asociadas a la neumonía fueron: edad $(p=0.05)$, hospitalización previa $(p=0.03)$ y enfermedad respiratoria previa $(p=0.002)$. Es decir tener entre 4 a 5 años y tener una hospitalización y enfermedad respiratoria previa se asociaron con la neumonía. El resto de asociaciones no fueron significativas. Respecto al análisis de modelo no ajustado (crudo) las características que se asociaron estadísticamente con la neumonía fueron: la edad $(p=0.01)$, hospitalización $(p=0.01)$ y enfermedad respiratoria previa $(p=0.02)$. Los niños con edades entre 4 a 5 años tenían 1.2 veces más veces a presentar neumonía en comparación de los que tienen $\leq 1$ año (OR: 2.2; IC $95 \%$ : 1.2 - 4.1). Por otra parte, los niños con una hospitalización previa tienen 0.8 veces más riesgo a presentar neumonía que los que no se han hospitalizado antes (OR: 1.8; IC $95 \%$ : 1.1-3.02). Asimismo, aquellos que han tenido una enfermedad respiratoria previa presentan 2.8 veces más riesgo de neumonía en relación de los niños que no han tenido (OR: 3.8; IC $95 \%$ : 1.6-8.9).

\section{DISCUSIÓN}

Entre los 293 niños del periodo estudiado se evidenció que el $45.7 \%$ presentó neumonía. Además, las características de la madre que se asociaron con la neumonía fueron presentar ocupación laboral, y como factor protector fue brindar lactancia materna exclusiva. Respecto a las características del menor para presentar neumonía fueron: tener cuatro a cinco a años y tener una hospitalización y enfermedad respiratoria previas.

Brindar lactancia materna exclusiva fue un factor protector para que el niño menor de cinco años no presente neumonía. Estudios muestran resultados similares donde mencionan que no brindar LME se asocia a tener neumonía $(14,16-19)$,entre otras enfermedades respiratorias como bronquitis, bronquiolitis, IRA $(8,15,19)$ y asma (17).

Estudios evidencian que la LME reduce el riesgo de muertes por neumonías en niños menores de 2 años $(7,20,21)$. Esto se debe a que la leche materna contiene factores anti infecciosos (inmunoglobulina A secretoria (IgAs), glóbulos blancos, oligosacáridos y proteínas de suero) que 
Tabla 2. Análisis bivariado entre las características del menor asociados a la neumonía en niños menores de 5 años de una clínica de Lima, 2018

\begin{tabular}{|c|c|c|c|c|c|}
\hline Factores & Casos n (\%) & Controles n (\%) & $p$ & OR $(\mathrm{IC} 95 \%) \dagger$ & $\mathbf{p}$ \\
\hline \multirow[t]{4}{*}{ Edad } & & & 0.05 & & \\
\hline & $42(38.9)$ & $66(61.1)$ & & Referencia & - \\
\hline & $35(48.6)$ & $37(51.4)$ & & $1.5(0.8-2.7)$ & 0.20 \\
\hline & $33(58)$ & $26(42)$ & & $2.2(1.2-4.1)$ & 0.02 \\
\hline Peso al nacer (media \pm DS) & $3185.2 \pm 613.7$ & $3251.4 \pm 522.5$ & $0.36^{*}$ & $0.9(0.9-1)$ & 0.36 \\
\hline \multicolumn{3}{|l|}{ Hospitalización previa } & 0.03 & & \\
\hline No & $60(41.1)$ & $86(58.9)$ & & Referencia & - \\
\hline Sí & $54(55.7)$ & $43(44.3)$ & & $1.8(1.1-3.02)$ & 0.03 \\
\hline \multicolumn{3}{|c|}{ Enfermedad respiratoria previa } & 0.002 & & \\
\hline No & $15(37.5)$ & $25(62.5)$ & & Referencia & - \\
\hline Sí & $41(69.5)$ & $18(30.5)$ & & $3.8(1.6-8.9)$ & 0.002 \\
\hline \multicolumn{3}{|l|}{ Sexo } & 0.83 & & \\
\hline Masculino & $50(47.2)$ & $56(52.8)$ & & Referencia & - \\
\hline Femenino & $60(45.8)$ & $71(54.2)$ & & $0.9(0.6-1.6)$ & 0.83 \\
\hline \multicolumn{3}{|l|}{ Vacunas } & 0.815 & & \\
\hline Incompletas & $10(50)$ & $10(50)$ & & Referencia & \\
\hline Completas & $104(47.3)$ & $116(52.7)$ & & $0.9(0.4-2.2)$ & 0.82 \\
\hline \multicolumn{3}{|c|}{ Retraso de desarrollo psicomotriz } & 0.183 & & \\
\hline No & $108(45.9)$ & $127(54.1)$ & & Referencia & - \\
\hline Sí & $5(71.4)$ & $2(28.6)$ & & $2.9(0.56-15.5)$ & 0.20 \\
\hline
\end{tabular}

fortalecen el sistema inmunológico del lactante, (8, 22) indispensables para un mejor crecimiento y desarrollo de los niños y la prevención de enfermedades infecciosas que contribuyen con su morbilidad y mortalidad $(23,24)$. Según la OMS $(8,9,21)$ y el Fondo de las Naciones Unidas para la Infancia (UNICEF) estos beneficios son más efectivos si se brinda la lactancia materna exclusiva durante los 6 meses y no limitada parcialmente de tres a cuatro meses $(20,21)$.

La ocupación laboral de la madre se asoció significativamente con la presencia de neumonía en niños menores de cinco años. Son pocos los estudios actuales sobre esta relación causal, solo un estudio evidenció que la madre con un trabajo fuera de casa es un factor de riesgo para la neumonía en sus hijos (13). Actualmente se observa una creciente participación laboral femenina para complementar la renta familiar del hogar, pero esto conlleva a la madre a no tener un cuidado directo con su hijo y por ende decide en muchos casos llevarlo a guarderías. Un estudio evidenció que los niños que asistían a guarderías presentaban un riesgo mayor de hospitalización por infecciones respiratorias agudas en comparación con niños con cuidados en su domicilio (10). Esto podría deberse a la exposición de otros niños con enfermedades respiratorias y por una alimentación inadecuada. Por otro lado, las madres tienen obstáculos para dar lactancia materna exclusiva debido a su trabajo porque no se cumple la licencia de horas de lactancia y no hay lactarios en su establecimiento laboral, etc. (25). Teniendo en cuenta que es un factor protector contra la neumonía en niños menores de 5 años, es la LME durante los 6 primeros meses de vida (12).

Respecto a las características del menor en el estudio se encontró que tener entre 4 a 5 años de edad fue un factor de riesgo para presentar neumonía. Estudios confirman el hallazgo evidenciando que la neumonía afecta más a niños entre 3 y 5 años $(17,26)$. En otro estudio se 
encontró que las edades más frecuentes de los niños que se hospitalizan fueron de 2 a 5 años (27). Esto es posible porque los niños de esta edad se encuentran expuestos a múltiples factores ambientales como biológicos (infecciones respiratorias), químicos orgánicos e inorgánicos (metales pesados, plaguicidas, etc.) y psicosociales (tabaquismo en su comunidad) (13). Además, por un intercambio con el medio social (nidos, escuelas y círculos infantiles). Se debe tener en cuenta que los niños en edades preescolares debido a sus condiciones fisiológicas y las características de sus mecanismos defensivos (inmadurez del sistema inmunitario) se encuentran con predisposición de infecciones respiratorias (24).

Tener una hospitalización previa y causada por una enfermedad respiratoria fue un factor de riesgo para presentar neumonía. Estudios detallan que los niños que tienen neumonía han tenido hospitalizaciones previas por las mismas patologías $\mathrm{u}$ otras enfermedades respiratorias asociadas (25-27). Esto podría deberse que no hay seguimiento extrahospitalario de los niños que fueron dados de alta. Un estudio mostró que entre $7.3 \%$ y $9.8 \%$ de pacientes dentro de las 72 horas posteriores al alta presentaron síntomas compatibles con infecciones respiratorias, porque habían compartido un habitación con niños con estas patologías (27). Además, el hecho de que el niño haya presentado una enfermedad respiratoria previa conlleva a que los pulmones pueden quedar más susceptibles a ser afectados por una neumonía u otro tipo de IRA (10).

Entre las limitaciones principales se señala que no se pudieron medir otras características de interés como por ejemplo si la madre llevaba a su menor al control de crecimiento y desarrollo (CRED) y las características ambientales en el hogar: si la madre u otro miembro de la familia fuma dentro del hogar, hacinamiento, etc. Esto se debe a que el estudio fue retrospectivo y las historias clínicas no contaban con estos datos. Por esta razón se recomienda realizar futuros estudios teniendo en cuenta estas características y razones de la estancia hospitalaria prolongada en niños con neumonía.

\section{CONCLUSIONES}

Los datos del estudio evidencian que en la población estudiada hay una prevalencia del 45.7 $\%$ de neumonía. La lactancia materna exclusiva es factor protector, mientras que la ocupación laboral y las características del menor: tener 4 a 5 años de edad y tener una hospitalización y enfermedad respiratoria previas son factores de riesgo de la neumonía en los niños menores de 5 años.

A partir de estos hallazgos se recomienda que el personal de salud tenga en cuenta los mismos para brindar una adecuada campaña de prevención sobre la neumonía a las madres y otros cuidadores. Asimismo, el personal de enfermería debe brindar intervenciones que fomenten la lactancia materna exclusiva con previa supervisión a las madres para verificar $\mathrm{su}$ práctica correcta.

\section{REFERENCIAS BIBLIOGRÁFICAS}

1. Organización Mundial de la Salud (OMS). Neumonía, datos y cifras [internet]. Ginebra, Suiza: OMS; 2018 [citado el 20 de julio de 2018]. Disponible en: 3 http://www.who.int/es/news room/detail/11-04-2018-who-andunicef-is sue-new-guidance-topromote-breastfeeding-in-healthfacilities-globally.

2. Rudan I, Boschi C, Biloglav K, Mulholland K. Epidemiología y etiología de la neumonía en la niñez. Boletín de la Organización 4. Organización Panamericana de la Mundial de la Salud. 2008;86(1): 321-416. Salud (OPS). La neumonía es la causa principal de muerte de niños [internet]. Washington, Estados Unidos: OPS y OMS; 2018 [citado el 22 de julio de 2018]. Disponible en

:.https://www.paho.org/uru/index. php?option=com_content $\& v i e w=a$ rticle\&id=460:la-neumonía-causaprincipal-muerte-ninos \&Itemid $=340$. 
5. Ministerio de Salud (MINSA.) 11. Alzate MC, Arango C, Castaño JJ, 16. Lamberti1 LM, Irena Zakarija I,

Número de episodios de neumonías. Perú 2013-2018 [internet]. Lima, Perú: MINSA [citado el 01 de agosto del 2018]. Disponible

en:http://www.dge.gob.pe/portal/d ocs/tools/teleconferencia/2018/SE 242018/01.pdf.

6. Padilla J, Rizo E, Medina MC. 12. Instituto Nacional de Estadística e Neumonías en niños en el Perú: Tendencias epidemiológicas, intervenciones y avances. Revista Médica Clínica Las Condes. 2017; 28(1)97-103.

7. Instituto Nacional de Estadística e Informática. Indicadores de Resultados de los Programas Presupuestales, Primer Semestre 2017 [internet]. Lima, Perú; 2017 [citado el 2 de agosto de 2018]. Disponible en: https://proyectos.inei.gob.pe/endes /images/Indicadores_Resultados_P PR_Primer_Semestre_2017.pdf

8. Pérez M, Fundora H, Notario M, Pérez R. Factores de Riesgo Inmuno Epidemiológicos en niños con infecciones respiratorias recurrentes. Cubana de Pediatría. 2011; 83(3):225-235.

9. Bedoya J, Benítez LP, Castaño JJ, Mejía O, et al. La lactancia materna y su relación con patologías prevalentes en la infancia en jardines infantiles de la ciudad de Manizales. Archivos de Medicina (Col). 2013; 14(1):2943.

10. Muñoz L P, Martínez JW Quintero AR, Lazo GI, Median GA. Características asociadas a las prácticas de la lactancia materna asistentes a tres. Revista Investigaciones Andina. 2005; 7(11):17-27.
Henao AM, Lozano MM, Muñoz $\mathrm{G}$, et al. Lactancia materna como factor protector para enfermedades prevalentes en niños hasta de 5 años de edad en algunas instituciones educativas de Colombia 2009. Colomb Obstet Ginecol. 2011; 62(1):57-63.

Informática. Indicadores de Resultados de los Programas Presupuestales, Primer Semestre 2017 [internet]. Lima, Perú; 2017 [citado el 2 de agosto de 2018]. Disponible en: https://proyectos.inei.gob.pe/endes /images/Indicadores_Resultados_P PR_Primer_Semestre_2017.pdf

13. Fonseca W, Kirkwood BR, Cesar G. Factores de riesgo para la años en Fortaleza, Brasil. Bulletin of the World Health Organisation 1996; 74(2):199-208

14. Organización Panamericana de la 20. Salud (OPS). La alimentación del lactante y del niño pequeño. Capítulo Modelo para libros de texto dirigidos a estudiantes de medicina y otras ciencias de la salud [internet]. Washington, Estados Unidos: OPS; 2010 [citado el 3 de agosto de 2018]. Disponible en http://apps.who.int/iris/bitstream/h andle/10665/44310/978927533094 4_spa.pdf;jses sionid=C5A2. Restrepo, Segura AM. Conocimientos y prácticas del cuidador como factor asociado a enfermedad respiratoria aguda en niños de 2 meses a 5 años. Invest Educ Enferm. 2010;29(1):19-27.

22. Macedo, CR. Factores de interrupción de la lactancia neumonía en niños menores de dos
Fischer CL, et al. Breastfeeding for reducing the risk of pneumonia morbidity and mortality in children under two: a systematic literature review and metaanalysis. Public Health. 2013;13( 3):2-8.

17. Juraci AC, Cesar GV, Barros F, Santos IS, Flores J. Impact of breast feeding on admission for pneumonia during postneonatal period in Brazil: nested casecontrol study. Bmj, 1999;318(7194):1316-1320.

8. Minagawa AT, Fujimori E, Teixeira CN. Breastfeeding: what changed after a decade? Revista latino-americana de enfermagem 2017;25(1)2-10.

19. Tamayo CM, Calderón S, Cunill S, Díaz D, Maren. Enfermedad neumocócica en pacientes menores de 5 años. MEDISAN 2018;22(8):946.

20. Organización Mundial de la Salud. La OMS y el UNICEF publican nuevas directrices para promover la lactancia materna en los establecimientos de salud de todo el mundo [internet]. Ginebra, Suiza: OMS; 2018 [citado el 2 de agosto de 2018]. Disponible en: http://www.who.int/es/news room/detail/11-04-2018-who-andunicef-is sue-new-guidance-topromote-breastfeeding-in-healthfacilities-globally

P, 21. Aguilar MJ, Baena L, Sánchez A. Beneficios inmunológicos de la leche humana para la madre y el niño: revisión sistemática Nutrición Hospitalaria. 2016;33(2):482-493

materna exclusiva en madres que acuden al Centro de Salud de 
Ñaña, Lima 2016 [tesis de licenciatura]. Lima: Universidad Peruana Unión; 2017.

hospitalizados. Arch Pediatr Urug. 2003;74(1):6-14.

23. Zambrano BM. Factores sociales que influyen en la neumonía en pacientes lactantes [tesis de licenciatura]. Guayaquil: Universidad de Guayaquil; 2018.

24. Pirez MM, Berrondo C, Giacometti M, Demiguel M Pascale I, Algorta G, et al. Neumonía bacteriana adquirida en 26. Rodríguez A, González I, Moré Y, la comunidad en niños
25. Martínez ME. Factores ambientales y su incidencia en la insuficiencia respiratoria aguda en menores de 5 años de la ciudadela Barrio Lindo Babahoyo Los Ríos periodo septiembre 2017 a febrero 2018 [tesis de licenciatura] Babahoyo: Universidad técnica de Babahoyo; 2018.

Vázquez M. Neumonía bacteriana adquirida en la comunidad en niños hospitalizados. Arch Pediatr Urug. 2003;74(1):6-14.

27. Flores JC, Riquelme P, Cerda J, Carrillo D, Matus MS, Araya G, Viviani T. Mayor riesgo de infecciones asociadas a atención en salud en niños con necesidades especiales hospitalizados. Revista chilena de infectología. 2014; 31(3):287-292. 